Check for updates

Cite this: RSC Adv., 2021, 11, 34669

Received 23rd August 2021

Accepted 16th October 2021

DOI: $10.1039 / \mathrm{d} 1 \mathrm{ra06375h}$

rsc.li/rsc-advances

\section{Vertically oriented mesoporous silica film modified fluorine-doped tin oxide electrode for enhanced electrochemiluminescence detection of lidocaine in serum $\dagger$}

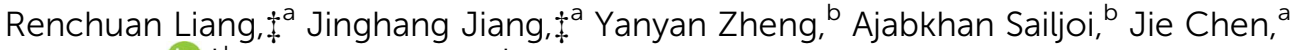 \\ Jiyang Liu (iD) $* b$ and Hongxue Li ${ }^{* a}$
}

Owing to a nanochannel-based enrichment effect and anti-fouling ability, highly ordered and vertically oriented mesoporous silica thin film (VMSF) modified electrodes have demonstrated their great potential in direct and highly sensitive analysis of complex samples. In this work, a VMSF modified fluorine-doped tin oxide (FTO) electrode (VMSF/FTO) is fabricated for enhanced electrochemiluminescence (ECL) analysis of lidocaine in serum. VMSF with good integrity and mechanical stability can be rapidly and conveniently grown on FTO in a few seconds at room temperature using an electrochemically assisted self-assembly (EASA) method. Due to the strong electrostatic attraction between the cationic ECL probe and negatively charged nanochannel, the VMSF/FTO electrode shows significant enrichment of tris(2,2bipyridine) ruthenium(II) $\left(\mathrm{Ru}(\mathrm{bpy})_{3}{ }^{2+}\right)$, leading to $\sim 10$ times enhancement of its $\mathrm{ECL}$ signal in comparison to the bare FTO electrode. Lidocaine, an anesthetic and antiarrhythmic drug, can act as the co-reactant of $\mathrm{Ru}(\mathrm{bpy})_{3}{ }^{2+}$ and promote its ECL signal. Sensitive ECL detection of lidocaine is achieved by the sensor in a wide linear range from $10 \mathrm{nM}$ to $50 \mu \mathrm{M}$ with a low limit-of-detection (LOD) of $8 \mathrm{nM}$. Combined with the antifouling ability of VMSF, the VMSF/FTO electrode also realizes the accurate and rapid analysis of lidocaine in real serum samples.

\section{Introduction}

Highly ordered solid-state nanofilms have recently demonstrated great potential in (bio)sensing, molecular separation, energy conversion, catalysis, delivery, and nanofluids owing to their unique advantages of adjustable nanopores, high specific surface area, controlled molecular transportation, and easy integration ability. ${ }^{1-3}$ Amongst such films, the vertically oriented mesoporous silica film (VMSF) is attractive as a mesoporous nanofilm prepared using self-assembled surfactant micelles as templates. ${ }^{4-6}$ VMSF consists of nanochannel array perpendicular to the supporting electrode, which has uniform channel size (generally $2 \sim 3 \mathrm{~nm}$ in diameter) and high density (up to 75000 per $\left.\mu \mathrm{m}^{2}\right) .^{7-10}$ The high density of nanochannel and nanosized thickness ensure efficient diffusion and mass transfer from solution to the supporting electrode. More importantly, VMSF nanochannel has permselectivity based on

\footnotetext{
${ }^{a}$ Guangxi Medical University Cancer Hospital, Guangxi Medical University, 71 Hedi Road, Nanning 530021, PR China.E-mail: lihongxue1616@163.com

${ }^{b}$ Department of Chemistry, Zhejiang Sci-Tech University, 928 Second Avenue, Xiasha Higher Education Zone, Hangzhou, 310018, PR China.E-mail: liujy@zstu.edu.cn $\dagger$ Electronic supplementary information (ESI) available. See DOI: 10.1039/d1ra06375h

\$ The two authors contributed equally.
}

size, charge and hydrophobicity of molecules because its ultrasmall aperture is close to the Debye length or electric double layer thickness. ${ }^{11-13}$ On the one hand, VMSF has significant size exclusion effect and can effectively exclude large particles (e.g. cells) and macromolecules (e.g. proteins), showing outstanding anti-fouling ability. On the other hand, the surface of VMSF is negatively charged under conventional $\mathrm{pH}$ conditions because of the ionization of $\mathrm{Si}-\mathrm{OH}$ groups $\left(\mathrm{p}_{\mathrm{a}} 2 \sim 3\right)$. Thus, VMSF displays remarkable enrichment towards positively charged molecules (especially larger organic cations), producing significant signal amplification. At the same time, electrostatic repulsion to negatively charged molecules leads to good anti-interference properties. Therefore, VMSF shows great potential in modifying electrode to fabricate sensors for direct detection of complex samples.

Until now, VMSF can be conveniently prepared using Stöber solution growth or electrochemically assisted self-assembly (EASA) method., ${ }^{\mathbf{4 1 4}, 15}$ The supporting electrode to equip VMSF are mainly divided into two categories. One is indium tin oxide (ITO) electrode, the other is carbon or metal electrode. Owing to covalent bonding between VMSF and ITO surface by forming -Si-O-In or -Si-O-Sn bonds, VMSF can be stably equipped on ITO substrate and exhibits high mechanical stability. However, indium is a rare element with low storage in nature and ITO is 
of high price. Though carbon or metal electrodes have the advantages of diverse forms (e.g., glassy carbon, graphene, graphite, carbon fibre, gold, platinum, etc.) and low detection overpotential, VMSF on these materials is easy to fall off because of weak adhesion. To improve the stability of VMSF, molecular glue (e.g., 3-aminopropyltrimethoxysilane, APTES) or nanomaterial adhesive layer is essential, resulting in complex operation process. ${ }^{\mathbf{1 6 - 1 8}}$ Facile and stable equipment of VMSF on efficient and cheap electrode is highly desirable to further explore the applications of VMSF-based sensors.

Electrochemiluminescence (ECL) is a luminescence caused by electron transfer and chemical reaction of reactants at the electrode surface under the excitation of electrochemical process. ${ }^{19-25}$ Compared with other luminescence methods (e.g., fluorescence and chemiluminescence), ECL technique possesses the advantages of zero background and high signalto-noise, facilitating highly sensitive detection. At the same time, ECL reaction is strictly controlled by electrochemical process and occurs in the diffusion layer near the electrode surface, providing controllable reaction and high sensitivity. Therefore, ECL has been a powerful analysis tool and widely applied in a wide range of applications (e.g., immuno-assay, drug analysis, etc.). Among various ECL luminophores, tris(2,2-bipyridine) ruthenium(II) $\left(\mathrm{Ru}(\mathrm{bpy})_{3}{ }^{2+}\right)$ is widely used because of its high ECL efficiency. ${ }^{26-29}$ However, its high price increases the cost of the corresponding analysis. Since $\mathrm{Ru}(\mathrm{bpy})_{3}{ }^{2+}$ is a large organic cation, the negatively charged VMSF nanochannels can effectively enrich $\mathrm{Ru}(\mathrm{bpy})_{3}{ }^{2+}$, which significantly increases the concentration of ECL luminophores on electrode surface, leading to high detection sensitivity and low usage of $\mathrm{Ru}(\mathrm{bpy})_{3}{ }^{2+} \cdot{ }^{12,13}$

In this work, we demonstrate facile equipment of verticallyordered mesoporous silica-nanochannel film on cheap fluorine-doped tin oxide (FTO) electrodes, which enable sensitive and direct ECL detection of lidocaine in serum. As shown in Fig. 1, VMSF is easily and rapidly grown on the surface of FTO electrode (VMSF/FTO) by EASA method using SM as template. After SM removal, the open nanochannels with negative charge are able to enrich $\mathrm{Ru}(\mathrm{bpy})_{3}{ }^{2+}$ through the strong electrostatic attraction. Even in absence of co-reactant, the ECL intensity of $\mathrm{Ru}(\mathrm{bpy})_{3}{ }^{2+}$ can be enhanced nearly 10 times. As a proof-of-

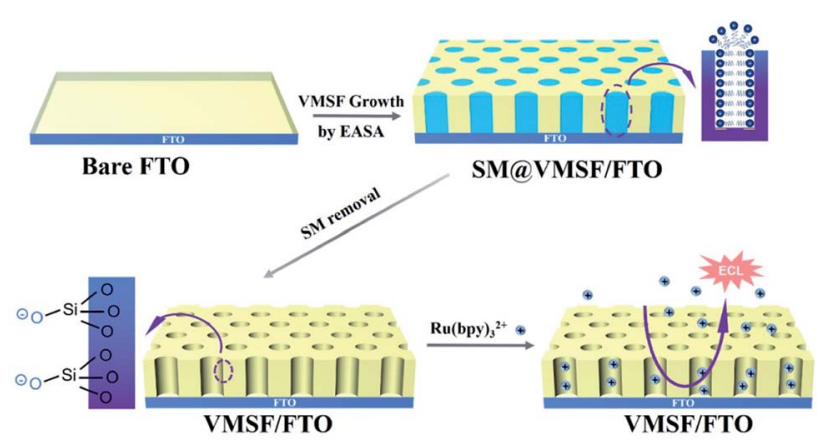

Fig. 1 Illustration for the preparation of VMSF/FTO using EASA method and the enrichment of $\mathrm{Ru}(\mathrm{bpy})_{3}{ }^{2+}$ on negatively charged nanochannels. demonstration, sensitive and rapid ECL analysis of lidocaine in real serum samples is realized by VMSF/FTO in combination of the antifouling ability of VMSF.

\section{Experimental}

\subsection{Materials and reagents}

Tris(2,2-bipyridine)dichlororuthenium(II) hexahydrate (Ru(bpy) ${ }_{3}$ $\mathrm{Cl}_{2} \cdot 6 \mathrm{H}_{2} \mathrm{O}$ ) was purchased from Sigma-Aldrich (USA). Tetraethoxysilane (TEOS), lidocaine, cetyltrimethylammonium bromide (CTAB), potassium ferricyanide $\left(\mathrm{K}_{3}\left[\mathrm{Fe}(\mathrm{CN})_{6}\right]\right), \mathrm{NaH}_{2} \mathrm{PO}_{4}, \mathrm{Na}_{2} \mathrm{HPO}_{4}$ were obtained from Aladdin (China). The phosphate buffer solution (PBS, $0.01 \mathrm{M}$, pH 7.0) was prepared by mixing $\mathrm{NaH}_{2} \mathrm{PO}_{4}$ and $\mathrm{Na}_{2} \mathrm{HPO}_{4}$. FTO glass ( $<10 \Omega$ per square) was obtained from Zhuhai Kaiwo Optoelectronics (China). Human blood serum was obtained by Hangzhou Institute of Occupational Diseases (Hangzhou, China). All other chemicals are of analytical grade and used without further treatment. Ultrapure water $(18.2 \mathrm{M} \Omega \mathrm{cm})$ was used to prepare all aqueous solutions.

\subsection{Characterizations}

The size, length, and uniformity of VMSF nanochannels were characterized by transmission electron microscopy (TEM). Before characterization, VMSF was gently stripped from the electrode surface and sonicated in ethanol for $5 \mathrm{~min}$. The dispersed sample was dropped on the supporting copper mesh to obtain top-view and cross-sectional TEM images of VMSF on JEM-2100 microscope (JEOL Ltd., Japan). The applied acceleration voltage is 200 $\mathrm{kV}$. Electrochemical measurements including cyclic voltammetry and electrochemical impedance spectroscopy were carried out on PGSTAT302N electrochemical workstation (AutoLab, Switzerland). The ECL measurement was performed on MPI-E II analysis system (Xi'an remex Analytical Instrument Co., Ltd., China).

\subsection{Preparation of VMSF/FTO electrode}

As reported, VMSF was grown on FTO by EASA method. ${ }^{4,29} \mathrm{~A}$ three-electrode system was used with bare FTO as the working electrode and platinum sheet $(\mathrm{Pt}, 1 \mathrm{~cm} \times 1 \mathrm{~cm})$ as the counter electrode. $\mathrm{Ag} / \mathrm{AgCl}$ electrode (saturated $\mathrm{KCl}$ solution) was applied as the reference electrode. Before use, FTO $(0.5 \mathrm{~cm} \times 1$ $\mathrm{cm}$ ) was firstly washed with $\mathrm{NaOH}$ aqueous solution (1 M) and then successively sonicated in acetone, ethanol, and ultrapure water, respectively. To grow VMSF, the precursor solution was prepared with TEOS $(13.6 \mathrm{mmol})$, CTAB $(4.35 \mathrm{mmol})$ in the mixture of $\mathrm{NaNO}_{3}(0.1 \mathrm{M}, 20 \mathrm{~mL})$ and ethanol $(20 \mathrm{~mL})$. After $\mathrm{pH}$ was adjusted to 3.0 with $\mathrm{HCl}$ solution $(6 \mathrm{M})$, the solution was stirred at room temperature for $2.5 \mathrm{~h}$ for pre-hydrolysis. Then, bare FTO was immersed in the precursor solution and applied a constant current of $-350 \mu \mathrm{A}$ for $10 \mathrm{~s}$. After the growth of VMSF, the obtained electrode with surfactant micellar (SM) inside the nanochannels (SM@VMSF/ITO) was quickly taken out and thoroughly washed with ultrapure water. After drying with nitrogen $\left(\mathrm{N}_{2}\right), \mathrm{SM@VMSF/ITO} \mathrm{electrode} \mathrm{was} \mathrm{aged} \mathrm{at} 120{ }^{\circ} \mathrm{C}$ overnight followed with SM removal by stirring in $0.1 \mathrm{M} \mathrm{HCl} /$ ethanol for $5 \mathrm{~min}$. The obtained electrode with open nanochannels was termed as VMSF/FTO. 


\subsection{ECL detection of lidocaine}

For ECL detection, PBS (0.1 M, pH 7.0) containing Ru(bpy $)_{3}{ }^{2+}$ $(10 \mu \mathrm{M})$ was used as the supporting solution. To detect lidocaine, VMSF/FTO was immersed in the supporting solution to enrich $\mathrm{Ru}(\mathrm{bpy})_{3}{ }^{2+}$ for $5 \mathrm{~min}$ with stirring. Then, different concentrations of lidocaine were added and the corresponding ECL signals were recorded during cyclic voltammetric scanning. The potential range of cyclic voltammetric scan is $0-1.25 \mathrm{~V}$ with a scan rate of $100 \mathrm{mV} \mathrm{s}^{-1}$. The voltage of photomultiplier tube in ECL detection was set as $600 \mathrm{~V}$. Standard addition recovery method was applied to detection lidocaine in serum. Specifically, serum with different concentrations of lidocaine was added into the supporting solution and then was detected using the same procedure.

\section{Results and discussion}

\subsection{Preparation and characterization of VMSF/FTO electrode}

As an attractive mesoporous thin film, VMSF can be grown through Stöber solution growth, EASA method, two phase growth method, and magnetically induced orientation, etc. ${ }^{\mathbf{1 4 , 3 0}}$ Amongst, the EASA method realizes convenient growth of VMSF in a few seconds at room temperature. As illustrated in Fig. 1, VMSF/FTO electrode is prepared by EASA method. Cationic CTAB is applied as the surfactant template and TEOS is the silica precursor. The growth mechanism lies in kinetically controlled interface nucleation/growth by a local $\mathrm{pH}$ increase on the surface of electrode generated from the reduction of protons and water molecules under cathodic potentials. This electrochemistry-induced $\mathrm{pH}$ gradient facilitates the self-assembly of CTAB semi-micelles on FTO surface as well as the polycondensation of TEOS around the surfactant micelle (SM). After VMSF growth, the VMSF modified electrode filled with SM in the nanochannel (SM/VMSF/FTO) is obtained. After destroying and removing $\mathrm{SM}$ with $\mathrm{HCl} / \mathrm{ethanol}$, VMSF/FTO with open nanochannels is obtained, which offers potential for electrostatic enrichment of positively charged molecules owing to negatively charged nanochannels from the ionization of $\mathrm{Si}-\mathrm{OH}$ groups.

Fig. 2 illustrates the top-view and cross-sectional TEM images of VMSF stripped from the electrode surface. As shown in the top-view image (Fig. 2A), VMSF has uniformly distributed nanopore structure with no cracks in the observed submicron range. The corresponding TEM image with high magnification (inset in Fig. 2A) reveals highly ordered arrange of nanopore (bright spots in the image) with a diameter of 2-3 $\mathrm{nm}$.
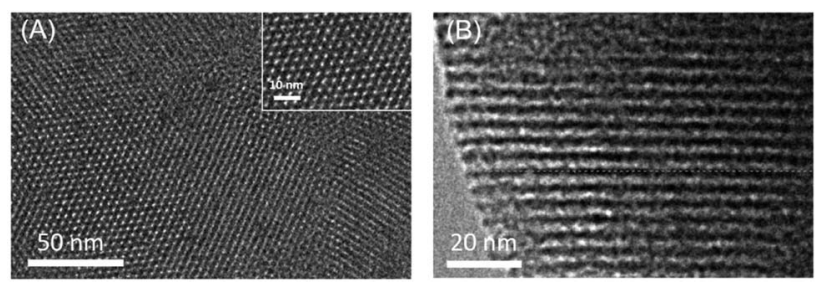

Fig. 2 (A) Top-view and (B) cross-sectional TEM images of VMSF. Inset in $(A)$ is TEM image at high magnification.

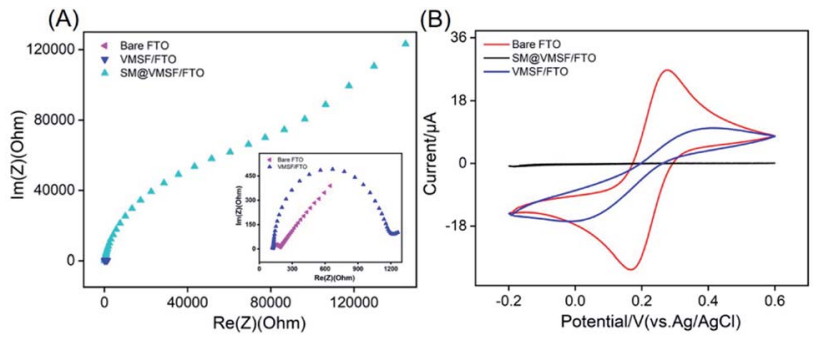

Fig. 3 EIS (A) and cyclic voltammetric (B) curves of bare FTO, SM@VMSF/FTO and VMSF/FTO in $0.1 \mathrm{M} \mathrm{KCl}$ solution containing $2.5 \mathrm{mM} \mathrm{Fe}(\mathrm{CN})_{6}{ }^{3-/ 4-}$. The inset in $(\mathrm{A})$ is the magnified EIS plots of FTO and VMSF/FTO. The scan rate in (B) is $50 \mathrm{mV} \mathrm{s}^{-1}$.

Nanochannels with thickness of $\sim 100 \mathrm{~nm}$ is easily identified from the cross-sectional TEM image (Fig. 2B). Thus, VMSF displays ultrasmall and high density nanochannels perpendicular to FTO electrode, which possesses unique advantages including high surface area and fast mass transfer.

The electrochemical behaviour of VMSF/FTO electrode was investigated by electrochemical impedance spectroscopy and cyclic voltammetry using $\mathrm{Fe}(\mathrm{CN})_{6}{ }^{3-/ 4-}$ as electrochemical probe. Bare FTO and SM/VMSF/FTO electrodes were also studied for comparison. As shown in Fig. 3A, bare FTO electrode has a very small electron transfer resistance $\left(R_{\mathrm{ct}}\right)$, indicating good electron transfer properties. When VSMF is grown and SM is contained in the nanochannel, the obtained SM/VMSF/FTO has significantly increased $R_{\mathrm{ct}}$, which is more than 400 times larger than that of bare FTO. Obviously, SM in the nanochannel totally hinders the diffusion of the electrochemical probe from the solution to the supporting electrode. When SM is removed, VMSF/FTO with open nanochannel displays slightly higher $R_{\mathrm{ct}}$ compared with bare FTO. This may be attributed to the electrostatic repulsion of negative VMSF nanochannels towards negative redox probes. The hindered probe diffusion by SM can also be verified through cyclic voltammetric curve (Fig. 3B). In comparison with bare FTO, almost no redox signal is observed on SM/VMSF/FTO, indicating an intact VMSF without crack. Despite permeable nanochannels, VMSF/FTO still exhibits lower signals compared with bare FTO, further proving the electrostatic repulsion of nanochannels to electrochemical probes. In addition, the redox peak of $\mathrm{Fe}(\mathrm{CN})_{6}{ }^{3-/ 4-}$ on $\mathrm{VMSF} /$ FTO electrode is almost unchanged when the electrode is stored in the refrigerator for one month, indicating stable VMSF modification on FTO. This may be attributed to the formation of $\mathrm{Si}-\mathrm{O}-\mathrm{Sn}$ bonds between VMSF and FTO.

\subsection{Enhanced ECL of Ru(bpy $)_{3}{ }^{2+}$ on VMSF/FTO electrode}

VMSF is expected to significantly enrich positively charged molecules (especially large organic cations) due to its high specific surface area and negative charge of nanochannels. As a proof-of-demonstrations, the most commonly used ECL probe, $\mathrm{Ru}(\mathrm{bpy})_{3}{ }^{2+}$ is chosen as the signal indicator because of its high ECL efficiency and positively charged characteristics. As revealed in Fig. 4A, the VMSF/FTO electrode exhibits significantly large anodic current signal at potentials higher than 
(A)

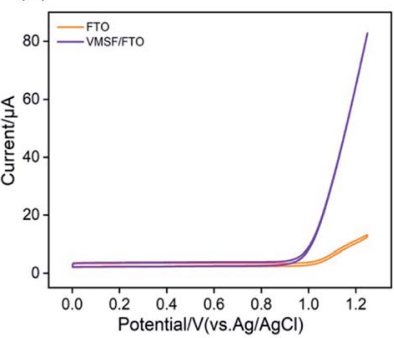

(B)

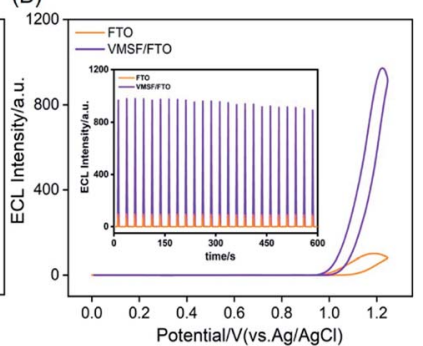

Fig. 4 Cyclic voltammetric curves (A) and ECL curves (B) obtained at bare FTO or VMSF/FTO in $0.1 \mathrm{M}$ PBS $(\mathrm{pH}$ 7) containing $10 \mu \mathrm{M}$ $\mathrm{Ru}(\mathrm{bpy})_{3}{ }^{2+}$. Inset in (B) is the $\mathrm{ECL}$ intensity during continuous cyclic voltammetric scan.

$\sim 0.9 \mathrm{~V}$ as compared with bare FTO electrode. At the same time, the corresponding ECL intensity of $\mathrm{Ru}(\mathrm{bpy})_{3}{ }^{2+}$ is $\sim 10$ times higher than that on bare FTO, suggesting VMSF/FTO can significantly enhance the ECL signal of $\mathrm{Ru}(\mathrm{bpy})_{3}{ }^{2+}$. This is mainly ascribed to the high-efficient enrichment of $\mathrm{Ru}(\mathrm{bpy})_{3}{ }^{2+}$ by the nanochannels, which increases its microregion concentration on the electrode surface, resulting in amplification of ECL intensity. As depicted in inset of Fig. 4A, the ECL intensity under continuous cyclic voltammetric scans shows a low relative standard deviation (RSD, 3.4\%), indicating good stability.

The charge characteristics and molecular permeability of VMSF nanochannels are significantly affected by ionic strength and $\mathrm{pH}$ of the supporting PBS. To obtain the highest enrichment effect of $\mathrm{Ru}(\mathrm{bpy})_{3}{ }^{2+}$, ionic strength and $\mathrm{pH}$ of the supporting PBS are investigated. A neutral PBS ( $\mathrm{pH} 7.0)$ with different ionic strengths is used. As shown in Fig. 5A, ECL intensity of $\mathrm{Ru}(\mathrm{bpy})_{3}{ }^{2+}$ in $0.01 \mathrm{M}$ PBS is the highest. At low ionic strength (the buffer concentration is less than $0.1 \mathrm{M}$ ), the electrical double layer (EDL) trends to overlap in the ultrasmall space of nanochannel ( $\sim 2 \mathrm{~nm}$ in diameter). In this case, VMSF nanochannels are forced to attract counter ions to decay electrostatic surface potential, leading to permselectivity of nanochannels with selective permeability of cationic ions and repelling of anions. ${ }^{31}$ In other words, low ionic strength facilitates the enrichment of $\mathrm{Ru}(\mathrm{bpy})_{3}{ }^{2+}$. As $0.001 \mathrm{M}$ PBS might lead to low conductivity, 0.01 M PBS with the highest ECL signal is chosen for further investigation. ECL signals at different $\mathrm{pH}$ are shown in Fig. 5B. When $\mathrm{pH}$ increases from 4 to 7 , the ECL strength of $\mathrm{Ru}(\mathrm{bpy})_{3}{ }^{2+}$ on VMSF/FTO electrode increases,
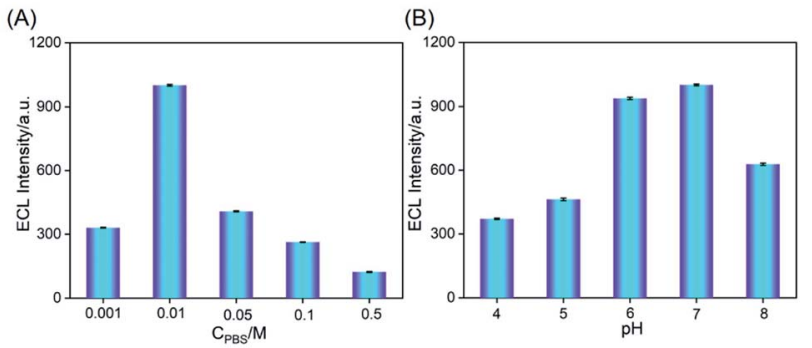

Fig. 5 ECL intensity obtained on VMSF/FTO in different concentrations (A) or $\mathrm{pH}$ (B) of PBS containing $10 \mu \mathrm{M} \mathrm{Ru}(\mathrm{bpy})_{3}{ }^{2+}$.

resulting from the increase of negative charge density of nanochannels due to the increased ionization of silicon hydroxyl at high $\mathrm{pH}$. The weak decrease of ECL signal at $\mathrm{pH} 8$ may be attributed to the decreased stability of silica under alkaline conditions. Thus, pH 7 is applied in further detection.

\subsection{Sensitive ECL detection of lidocaine}

Lidocaine (2-diethylamino- $N$-(2,6-dimethylphenyl)acetamide, abbreviated as Lid), as an anesthetic and antiarrhythmic drug, has obvious biphasic effects of excitation and inhibition on the central nervous system. When the blood concentration is low, analgesia, drowsiness and pain threshold increase, while the effect or toxicity increased with the increase of dose and the subtoxic concentration had anticonvulsant effect. ${ }^{26}$ Thus, sensitive detection of lidocaine in blood is of great significance. In comparison with the developed techniques for the detection of lidocaine including electrochemistry, fluorescence, and chemiluminescence, ECL sensing has great potential for sensitive detection due to the advantages of low background, high signalto-noise, and wide dynamic range.

As revealed in Fig. 6A, significant ECL increase of Ru(bpy $)_{3}{ }^{2+}$ is observed in presence of lidocaine, indicating that lidocaine can act as the co-reactant to promote the ECL of $\mathrm{Ru}(\mathrm{bpy})_{3}{ }^{2+}$. A possible mechanism of the ECL enhancement is shown in Fig. 6B. At high potential, $\mathrm{Ru}(\mathrm{bpy})_{3}{ }^{2+}$ is oxidized to $\mathrm{Ru}(\mathrm{bpy})_{3}{ }^{3+}$. At the same time, lidocaine is oxidized to its cationic free radicals, which produces a high-energy intermediate with strong reduction ability through deprotonation. Then, this intermediate reduces $\mathrm{Ru}(\mathrm{bpy})_{3}{ }^{3+}$ to form the excited state $\left[\mathrm{Ru}(\mathrm{bpy})_{3}{ }^{2+}\right]^{*}$, which produces ECL when returns to the ground state.

Fig. 6C shows the ECL signals obtained on VMSF/FTO electrode with different concentrations of lidocaine in PBS containing $\mathrm{Ru}(\mathrm{bpy})_{3}{ }^{2+}(10 \mu \mathrm{M})$. A good linear correlation is found
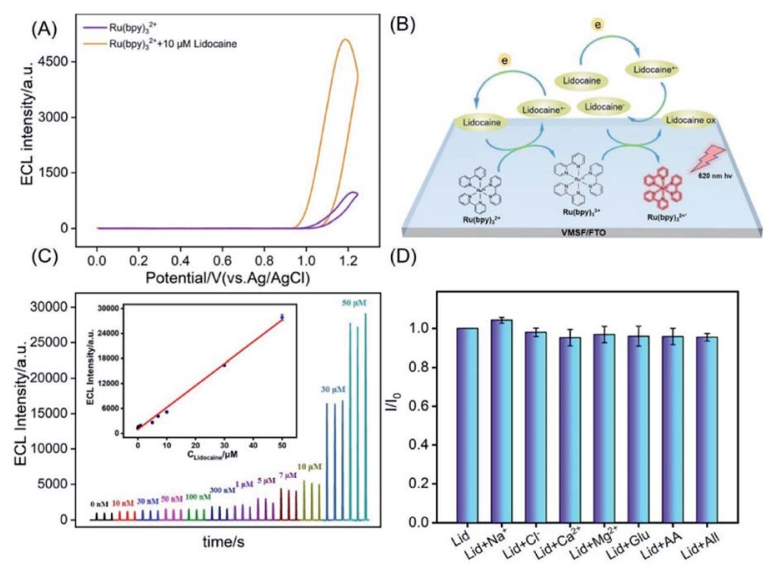

Fig. 6 (A) Cyclic voltammetric curves obtained at VMSF/FTO in $\mathrm{Ru}(\mathrm{bpy})_{3}{ }^{2+}$ solution $(10 \mu \mathrm{M}$ in $0.1 \mathrm{M}$ PBS, $\mathrm{pH} 7)$ in absence or presence of lidocaine. (B) The illustration for the possible ECL mechanism. (C) $E C L$ intensities with different concentrations of lidocaine. The inset is calibration curve for lidocaine determination. (D) The ECL intensities ratio $\left(/ / I_{0}\right)$ obtained on VMSF/FTO for detection of $20 \mu \mathrm{M}$ lidocaine (Lid) in the absence $(I)$ and presence $\left(I_{0}\right)$ of added interfering species $(1 \mathrm{mM})$. The concentration of the interfering species is $1 \mathrm{mM}$. 
Table 1 ECL determination of lidocaine in serum

\begin{tabular}{lclll}
\hline Sample & Added $(\mu \mathrm{M})$ & $\begin{array}{l}\text { Found } \\
(\mu \mathrm{M})\end{array}$ & $\begin{array}{l}\text { RSD } \\
(\%, n=3)\end{array}$ & $\begin{array}{l}\text { Recovery } \\
(\%)\end{array}$ \\
\hline Serum & 1.00 & 1.04 & 3.3 & 104.0 \\
& 5.00 & 5.33 & 1.1 & 106.6 \\
& 15.00 & 15.18 & 1.4 & 101.2
\end{tabular}

between the ECL intensity $\left(I_{\mathrm{ECL}}\right)$ and the concentration of lidocaine $\left(C_{\text {lidocaine }}\right)$ from $10 \mathrm{nM}$ to $50 \mu \mathrm{M}\left(I_{\mathrm{ECL}}=525 C_{\text {lidocaine }}{ }^{+}\right.$ $\left.983, R^{2}=0.995\right)$. The limit of detection (LOD) is determined as low as $8 \mathrm{nM}$. Lidocaine is usually synthesized by the reaction between $N$-(2,6-dimethylphenyl)chloroacetamide and diethylamine with $70-85 \%$ yield..$^{32}$ These two raw compounds might coexist in lidocaine. However, $N$-(2,6-dimethylphenyl)chloroacetamide cannot be used as a co-reactant of $\mathrm{Ru}(\mathrm{bpy})_{3}{ }^{2+}$. In case of diethylamine, it can be used as a co-reactant of $\mathrm{Ru}(\mathrm{bpy})_{3}{ }^{2+}$ due to the presence of secondary amine. However, the enhancement effect of ethylenediamine on ECL signal is not significant compared with compound with tertiary amine structure. Combining high purity and low linear detection range of lidocaine, the impurity of lidocaine will not affect the detection performance. Comparison between detection of lidocaine using different methods is given in Table S1 (ESI $\dagger)^{33-38}$ The LOD is lower than that obtained by an ECL sensor based on luminol-doped silica nanoparticles, ${ }^{33}$ or by capillary electrophoresis (CE) ${ }^{34}$ fluorescent sensor, ${ }^{35}$ highperformance liquid chromatography (HPLC), ${ }^{36,37}$ but slightly higher than that obtained using an ECL sensor based on 3D graphene paper electrode (GPE). ${ }^{38}$

\subsection{Detection selectivity and real sample analysis}

To detect lidocaine in serum, the selectivity of the detection is also evaluated using possible interference that exist in serum matrix. The tested interferences include ions $\left(\mathrm{Na}^{+}, \mathrm{Ca}^{2+}, \mathrm{Cl}^{-}\right.$, $\mathrm{Mg}^{2+}$ ), and biological small molecule such as glucose (Glu) or ascorbic acid (AA). As shown in Fig. 6D, one or the mixture of the above substances will not interfere with the detection of lidocaine, indicating high selectivity. The detection of lidocaine in serum is performed using standard addition method. As shown in Table 1, serum samples with artificially spiked lidocaine are examined with satisfactory recoveries ranged from $95.0 \%$ to $107.0 \%$. Thus, the developed sensor displays good reliability owing to the integration with VMSF, which offers high anti-fouling characteristics. The RSD for three measurements is less than $3.3 \%$, indicating high reproducibility.

\section{Conclusions}

In summary, we have developed a sensitive ECL sensor based on facile equipment of VMSF on FTO electrode, which enable rapid and convenient detection of lidocaine in serum. VMSF is easily grown on the surface of FTO at room temperature in a few seconds and exhibits high stability. Owing to significant enrichment of $\mathrm{Ru}(\mathrm{bpy})_{3}{ }^{2+}$ by nanochannels with negative charges, the developed VMSF/FTO electrode displays $\sim 10$ times enhanced ECL signal even without co-reactants. As lidocaine can act as co-reactor and remarkably increase the ECL of $\mathrm{Ru}(\mathrm{bpy})_{3}{ }^{2+}$, sensitive and direct detection of lidocaine in serum is realized by the sensor in combination with the anti-fouling ability of VMSF. It has been established that molecules with tertiary amine can act as efficient co-reactant of $\mathrm{Ru}(\mathrm{bpy})_{3}{ }^{2+}$. The developed sensor would also be sensitive enough for other active compounds with tertiary amine similar to lidocaine taking advantages of efficient enrichment of $\mathrm{Ru}(\mathrm{bpy})_{3}{ }^{2+}$ and detection using co-reactant mechanism. Owing to the easy fabrication, cheap electrode, and significantly enhanced ECL, the developed VMSF/FTO sensor may be extended when the nanochannels are further functionalized.

\section{Author contributions}

Renchuan Liang: data curation, Jinghang Jiang: data curation, Yanyan Zheng: data curation, Ajabkhan Sailjoi: data curation, Jie Chen: writing-reviewing and editing, Jiyang Liu: supervision, writing-reviewing and editing, Hongxue Li: supervision, writingoriginal draft preparation.

\section{Conflicts of interest}

There are no conflicts to declare.

\section{Acknowledgements}

The authors gratefully acknowledge the financial support from the National Natural Science Foundation of China (No. 81860512), Guangxi Natural Science Foundation (2018GXNSFAA138006), Guangxi Medical University Training Program for Distinguished Young Scholars, Guangxi med-ical high-level backbone talents " 139 " program training project, the Key Research and Development Program of Guangxi (AB18126032), and the Zhejiang Provincial Natural Science Foundation of China (LY20B050007).

\section{References}

1 I. Garbayo, A. Santiago, X. Judez, A. S. de Buruaga, J. Castillo and M. A. Muñoz-Márquez, Alumina nanofilms as active barriers for polysulfides in high-performance all-solid-state lithium-sulfur batteries, ACS Appl. Energy Mater., 2021, 4, 2463-2470.

2 X. Huang, L. Xie, X. Lin and B. Su, Permselective ion transport across the nanoscopic liquid/liquid interface array, Anal. Chem., 2016, 88, 6563-6569.

$3 \mathrm{~K}$. Chen, L. Yao and B. Su, Bionic thermoelectric response with nanochannels, J. Am. Chem. Soc., 2019, 141, 8608-8615.

4 T. Nasir, L. Zhang, N. Vila, G. Herzog and A. Walcarius, Electrografting of 3-aminopropyltriethoxysilane on a glassy carbon electrode for the improved adhesion of vertically oriented mesoporous silica thin films, Langmuir, 2016, 32, 4323-4332. 
5 H. Ding, W. Guo, P. Zhou and B. Su, Nanocage-confined electrochemiluminescence for the detection of dopamine released from living cells, Chem. Commun., 2020, 56, 8249-8252.

$6 \mathrm{~F}$. Yan and B. Su, Tailoring molecular permeability of nanochannel-micelle membranes for electrochemical analysis of antioxidants in fruit juices without sample treatment, Anal. Chem., 2016, 88, 11001-11006.

7 T. Nasir, G. Herzog, M. Hebrant, C. Despas, L. Liu and A. Walcarius, Mesoporous silica thin films for improved electrochemical detection of paraquat, ACS Sens., 2018, 3, 484-493.

8 S. L. Z. Jiokeng, I. K. Tonle and A. Walcarius, Aminoattapulgite/mesoporous silica composite films generated by electro-assisted self-assembly for the voltammetric determination of diclofenac, Sens. Actuators, B, 2019, 287, 296-305.

9 T. Nasir, A. Gamero-Quijano, C. Despas, M. Dossot, G. Herzog and A. Walcarius, Signal amplification by electro-oligomerisation for improved isoproturon detection, Talanta, 2020, 220, 121347.

10 J. Q. Liu, D. G. He and Q. Q. Liu, Vertically ordered mesoporous silica film-assisted label-free and universal electrochemiluminescence aptasensor platform, Anal. Chem., 2016, 88, 11707-11713.

11 L. Zhou, H. Ding, F. Yan, W. Guo and B. Su, Electrochemical detection of alzheimer's disease related substances in biofluids by silica nanochannel membrane modified glassy carbon electrodes, Analyst, 2018, 143, 4756-4763.

12 Z. Zhou, W. Guo, L. Xu, Q. Yang and B. Su, Two orders-ofmagnitude enhancement in the electrochemiluminescence of $\mathrm{Ru}(\mathrm{bpy})_{3}{ }^{2+}$ by vertically ordered silica mesochannels, Anal. Chim. Acta, 2015, 886, 48-55.

13 W. Guo, X. Lin, F. Yan and B. Su, Vertically ordered silica mesochannel modified bipolar electrode for electrochemiluminescence imaging analysis, ChemElectroChem, 2016, 3, 480-486.

14 Y. Guillemin, M. Etienne, E. Sibottier and A. Walcarius, Microscale controlled electrogeneration of patterned mesoporous silica thin films, Chem. Mater., 2011, 23, 5313-5322.

15 F. Yan, X. Lin and B. Su, Vertically ordered silica mesochannel films: electrochemistry and analytical applications, Analyst, 2016, 141, 3482-3495.

16 T. Nasir, N. A. Vodolazkaya, G. Herzog and A. Walcarius, Critical effect of film thickness on preconcentration electroanalysis with oriented mesoporous silica modified electrodes, Electroanalysis, 2019, 31, 202-207.

17 P. Zhou, L. Yao and B. Su, Fabrication, characterization, and analytical application of silica nanopore array-modified platinum electrode, ACS Appl. Mater. Interfaces, 2020, 12, 4143-4149.

18 F. Xi, L. Xuan, L. Lu, J. Huang, F. Yan, J. Liu, X. Dong and P. Chen, Improved adhesion and performance of verticallyaligned mesoporous silica-nanochannel film on reduced graphene oxide for direct electrochemical analysis of human serum, Sens. Actuators, B, 2019, 288, 133-140.
$19 \mathrm{Z}$. Liu, W. Qi and G. Xu, Recent advances in electrochemiluminescence, Chem. Soc. Rev., 2015, 44, 3117-3142.

$20 \mathrm{~W}$. Miao, Electrogenerated chemiluminescence and its biorelated applications, Chem. Rev., 2008, 108, 2506-2553.

$21 \mathrm{~L}$. Li, Y. Chen and J. J. Zhu, Recent advances in electrochemiluminescence analysis, Anal. Chem., 2017, 89, 358-371.

22 J. Zhang, R. Jin, D. Jiang and H. Y. Chen, Electrochemiluminescence-based capacitance microscopy for label-free imaging of antigens on the cellular plasma membrane, J. Am. Chem. Soc., 2019, 141, 10294-10299.

23 Q. Q. Huang, F. Luo and C. Y. Lin, Electrochemiluminescence biosensor for thrombin detection based on metal organic framework with electrochemiluminescence indicator embedded in the framework, Biosens. Bioelectron., 2021, 189, 113374.

24 Y. Xiao, S. H. Chen and S. M. Zhang, Mesoporous silicamediated controllable electrochemiluminescence quenching for immunosensor with simplicity, sensitivity and tunable detection range, Talanta, 2021, 231, 122399.

25 X. G. Sun, B. W. Li and C. Y. Tian, Rotational paper-based electrochemiluminescence immunodevices for sensitive and multiplexed detection of cancer biomarkers, Anal. Chim. Acta, 2018, 1007, 33-39.

26 X. M. Zhuang, X. Q. Gao, C. Y. Tian, D. L. Cui, F. Luan, Z. G. Wang, Y. Xiong and L. X. Chen, Synthesis of europium(III)-doped copper nanoclusters for electrochemiluminescence bioanalysis, Chem. Commun., 2020, 56, 5755-5758.

27 C. Y. Tian, L. Wang and F. Luan, A novel electrochemiluminescent emitter of europium hydroxide nanorods and its application in bioanalysis, Chem. Commun., 2019, 55, 12479-12482.

$28 \mathrm{~A}$. Abdussalam and $\mathrm{G}$. $\mathrm{Xu}$, Recent advances in electrochemiluminescence luminophores, Anal. Bioanal. Chem., 2021, DOI: 10.1007/s00216-021-03329-0.

29 A. Walcarius, E. Sibottier, M. Etienne and J. Ghanbaja, Electrochemically assisted self-assembly of mesoporous silica thin films, Nat. Mater., 2007, 6, 602-608.

30 Y. Liu, D. Shen, G. Chen, A. A. Elzatahry, M. Pal, H. Zhu, L. Wu, J. Lin, D. Al-Dahyan, W. Li and D. Zhao, Mesoporous silica thin membranes with large vertical mesochannels for nanosize-based separation, Adv. Mater., 2017, 29, 1702274.

31 W. Li, L. Ding, Q. Wang and B. Su, Differential pulse voltammetry detection of dopamine and ascorbic acid by permselective silica mesochannels vertically attached to the electrode surface, Analyst, 2014, 139, 3926-3931.

32 P. Josephson, V. Nykvist, W. Qasim, B. Blomkvist and P. Dinér, Student-driven development of greener chemistry inundergraduate teaching: synthesis of lidocaine revisited, J. Chem. Educ., 2019, 96, 1389-1394.

33 W. Qi, Y. Fu, H. He, M. Zhao, D. Wu, L. Qi, L. Hu and R. Li, Electrochemiluminescence resonance energy transfer for both "turn-off" detection of 2,4,6-trinitrophenol and "turnon" detection of lidocaine hydrochloride using luminol- 
doped silica nanoparticles, Sens. Actuators, B, 2019, 287, 445452.

34 Y. H. Yeh, W. C. Chen and S. Y. Chang, Surfactant-assisted dispersive liquid-liquid microextraction combined with field-amplified sample stacking in capillary electrophoresis for the determination of mexiletine and lidocaine, J. Sep. Sci., 2017, 40, 2406-2415.

35 Y. Zhang, Z. Y. Gao and W. Q. Zhang, Fluorescent carbon dots as nanoprobe for determination of lidocaine hydrochloride, Sens. Actuators, B, 2018, 262, 928-937.

36 B. Zargar and A. Hatamie, Hollow fiber liquid based microextraction combined with high-performance liquid- chromatography for the analysis of lidocaine in biological and pharmaceutical samples, Anal. Methods, 2014, 6, 25062511.

37 B. Chu, D. Lou and P. Yu, Development of an on-column enrichment technique based on C18-functionalized magnetic silica nanoparticles for the determination of lidocaine in rat plasma by high performance liquid chromatography, J. Chromatogr. A, 2011, 1218, 7248-7253.

38 Y. Han, Y. Fang, X. Ding, J. Liu, Z. Jin and Y. Xu, A simple and effective flexible electrochemiluminescence sensor for lidocaine detection, Electrochem. Commun., 2020, 116, 106760. 